\title{
Treatment of Pulmonary Embolism with Streptokinase. A Preliminary Report
}

\author{
G. A. H. MILLER,* D.M., M.R.C.P. ; R. V. GIBSON, † M.B., F.R.C.P. ; M. HONEY, † M.B., M.R.C.P. \\ G. C. SUTTON, $\ddagger$ M.B., M.R.C.P.
}

Brit. med. F., 1969, 1, 812-815

\begin{abstract}
Ummary : Nine patients with arteriographically proved pulmonary embolism have been treated by a 36-hour infusion of streptokinase. Satisfactory haemodynamic and arteriographic resolution was obtained in four patients with acute massive pulmonary embolism and in two with recent minor embolism. Little or no haemodynamic or arteriographic improvement was obtained in three patients with pulmonary thromboembolic disease of longer duration.
\end{abstract}

\section{Introduction}

Present uncertainty about the correct treatment of pulmonary embolism (British Medical fournal, 1968) derives from absence of a means of predicting, at the time of the acute episode, the chances of death or survival in any given patient. Moreover, not all patients who survive achieve complete resolution of emboli by natural lytic mechanisms or with the aid of anticoagulants (Goodwin et al., 1963). Embolectomy may be lifesaving, but it is a major procedure with a high mortality. On theoretical grounds, therefore, an alternative therapy is needed that is applicable both to acute massive pulmonary embolism and to patients with embolism of lesser severity in whom embolectomy seems unjustified. It is the purpose of this communication to report the results obtained by infusion of streptokinase in nine patients with pulmonary embolism of differing severity and duration.

\section{Methods}

Before treatment all patients were studied by cardiac catheterIzation, conventional techniques being used. Pressure measurements were referred to the mid-chest level. Single plane (anteroposterior) serial pulmonary arteriograms were obtained, contrast medium (Triosil 75 ; sodium metrizoate) being injected in a dose of $1 \mathrm{ml} . / \mathrm{kg}$. body weight by means of a pressure injector. The right brachial artery was cannulated with a fine polyethylene tube and samples from pulmonary and brachial arteries were analysed for oxygen saturation by means of a haemoreflector (Zijlstra and Mook, 1962). Oxygen consumption was measured, expired air being analysed for oxygen and carbon dioxide content with the micro-Scholander technique (Scholander, 1947). Cardiac output was expressed as $1 . / \mathrm{min}$./ sq.m. body surface area.

In all but one patient (Case 6) the pulmonary artery catheter was left in position and used for a 36-hour infusion of streptokinase (Kabikinase), usually in a dose of 600,000 i.u. for the first half-hour followed by 100,000 i.u./hour. Indwelling brachial and pulmonary artery catheters permitted repeated measurements of pulmonary artery pressure and arteriovenous ozygen difference during treatment. Repeated measurements of oxygen consumption permitted the calculation of cardiac

- Director, Cardiac Laboratories.

† Physician, Cardiac Department.

\$ Senior Registrar, Cardiac Department.

Brompton Hospital, London S.W.3. index and total pulmonary resistance during treatment. (Total pulmonary resistance is given by mean pulmonary artery pressure/cardiac index and expressed as units $\times$ sq. $\mathrm{m}$. body surface area.) Pulmonary arteriograms were repeated, sometimes on two occasions, at varying times during and after treatment, when further haemodynamic measurements were also made. Blood samples were taken for assay of coagulation factors and fibrinolysis before and at various times during treatment ; assays included fibrinogen, prothrombin time, and euglobulin lysis time. Hydrocortisone was given intramuscularly in a dose of $100 \mathrm{mg}$. before starting the infusion of streptokinase and all anticoagulants were withdrawn.

\section{Results \\ Acute Massive Pulmonary Embolism}

There were four patients, studied within 48 hours of the acute episode, in whom pulmonary arteriograms showed embolism involving at least half of the major pulmonary artery branches. Haemodynamic improvement was evident in all (Figs. 1-4) 12 to 15 hours after starting streptokinase infusion and was complete in three patients (Cases 1-3) at the time of the final study 48 hours, 8 days, and 28 days later respectively. In the fourth patient, with initial arteriographic evidence of pre-existing pulmonary thromboembolism, there was some residual pulmonary hypertension $(33 \mathrm{~mm}$. $\mathrm{Hg}$ systolic) at the time of the final study one month later. Arteriographic improvement was seen as early as 24 hours after starting treatment (Case 1) and was complete in two patients (Cases 1 and 2) when studied 48 hours and six days later respectively. In the remaining two patients considerable arteriographic improvement was present at 48 and 65 hours. Details of these patients are presented below and in Figs. 1-4.

Case 1.-Pulmonary arteriography performed 24 hours after the acute incident showed massive embolism involving all except the left upper lobe artery. The patient was restudied 24 hours after starting an infusion of streptokinase, which was continued for a total of 36 hours. At this time arteriography showed reduction in size of the filling defects which were still present. Pulmonary artery pressure, arteriovenous oxygen difference, cardiac index, and total pulmonary resistance were now normal. Six days after starting streptokinase a third pulmonary arteriogram showed complete disappearance of all filling defects and there had been a further slight fall in pulmonary artery pressure (Fig. 1).

Case 2.-Massive pulmonary embolism occurred 48 hours before the first study. A pulmonary arteriogram showed filling defects in branches of all pulmonary arteries. Since this patient was not critically ill it was decided to treat her with heparin alone for a further four days. A second study after a total of six days' treatment with heparin showed no haemodynamic or arteriographic improvement. Streptokinase was then infused into the pulmonary artery and continued for 36 hours. A third study performed 48 hours after starting streptokinase therapy showed complete haemodynamic and arteriographic resolution. Pulmonary artery pressure had fallen to normal levels within 18 hours of starting treatment (Fig. 2).

Case 3.-Pulmonary arteriography performed 48 hours after the acute incident showed a massive embolus at the bifurcation of the 
right pulmonary artery as well as emboli in distal branches of the left pulmonary artery. There was also collapse/consolidation of the right lower lobe distal to an occluded segmental pulmonary artery. A repeat study 48 hours after starting streptokinase therapy showed almost complete disappearance of the filling defects apart from occlusion of a segmental right lower lobe artery with reduction in volume of the corresponding lung segment. Pulmonary artery pressure was actually higher at this time than at the first study, though there had been a fall in the arteriovenous oxygen difference and total pulmonary resistance and a rise in cardiac index (Fig. 3). This patient still had some collapse of the right lower lobe when restudied 18 days after the initial event. At this time the pulmonary artery pressure was normal, as was the haemodynamic response to exercise (Fig. 3).

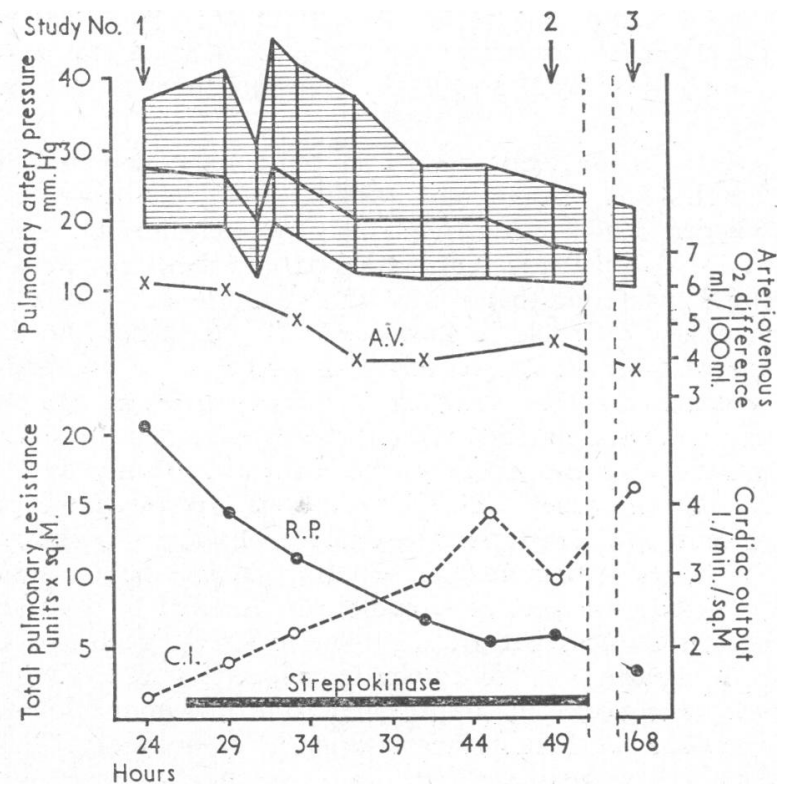

FIG. 1.-Case 1. A.V.=Arteriovenous oxygen difference. C.I. $=$ Cardiac index. R.P. = Total pulmonary resistance.

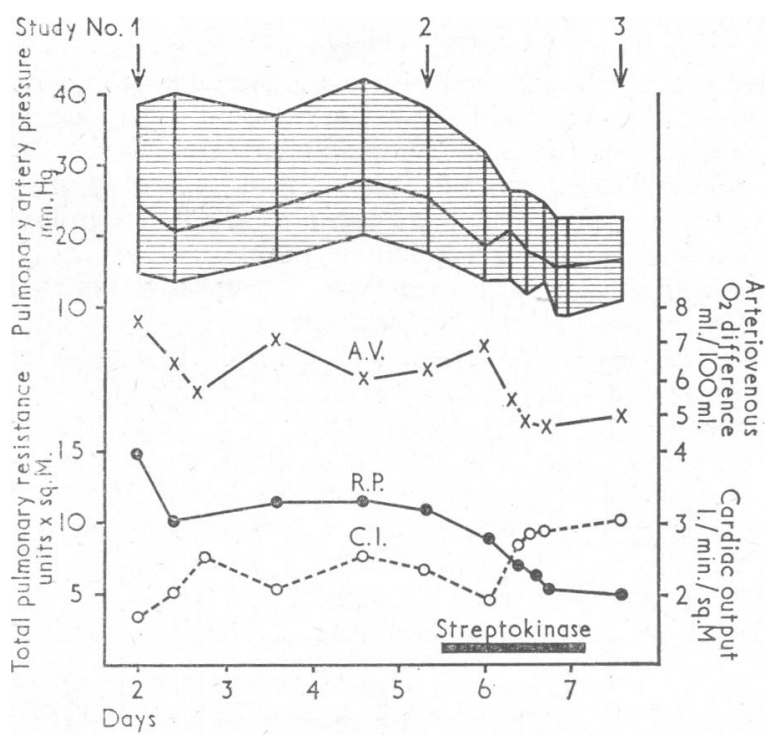

FIG. 2.-Case 2 .

Case 4.-Pulmonary arteriography performed less than 12 hours after the acute episode showed filling defects in all the branches of the pulmonary arteries. The arteriographic appearances suggested that not all of the embolism was recent and that there might have been previous thromboembolism involving the lower lobe arteries. Repeat study 65 hours after starting streptokinase showed considerable resolution of the emboli to the upper lobe arteries but again suggested old disease of the lower lobe arteries. At this time the pulmonary artery pressure was still raised, but the arteriovenous oxygen difference had fallen and the cardiac index become normal; the total pulmonary resistance had therefore fallen (Fig. 4). A third study one month later showed that pulmonary artery pressure was now lower though still marginally raised (Fig. 4). The pulmonary arteriogram did not show any further change.

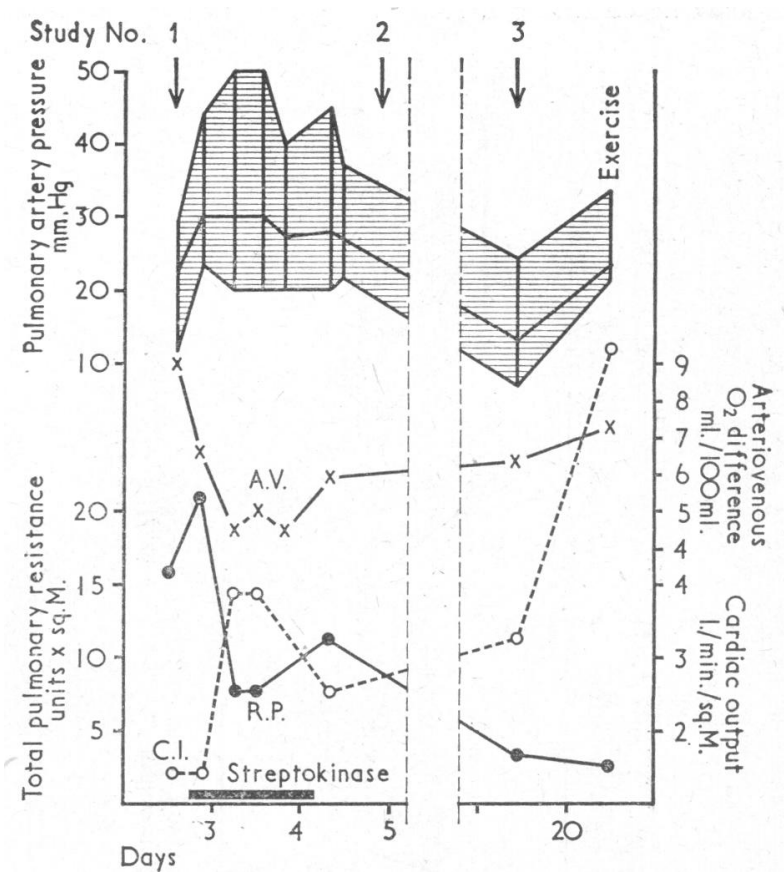

FIG. 3.-Case 3.
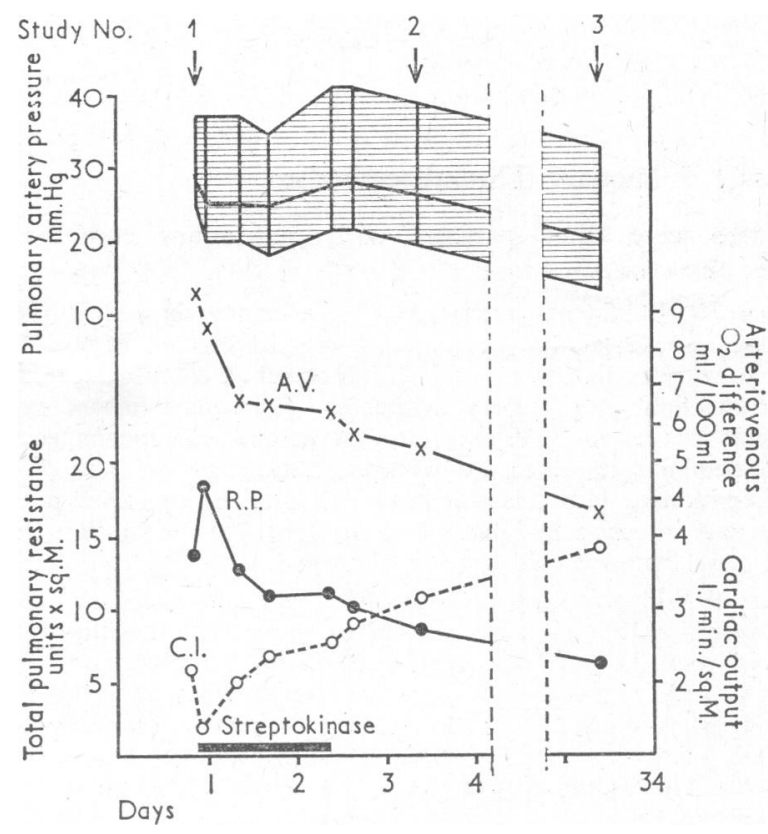

Fig. 4.-Case 4.

\section{Minor Recent Pulmonary Emboli}

There were two patients in whom pulmonary embolism had occurred six days and 12 hours before study respectively. Pulmonary arteriography showed involvement of less than half of the major pulmonary arterial branches and these patients were therefore classified as having minor pulmonary emboli. In one patient (Case 5) there was no haemodynamic disturbance, in the other (Case 6) there was pre-existing parenchymal lung disease and pulmonary artery pressure was raised (Fig. 5). Repeat study in this patient two days after starting a 30-hour infusion of streptokinase into the right atrium showed con- 
siderable haemodynamic improvement, though the pulmonary artery pressure $(33 \mathrm{~mm}$. $\mathrm{Hg}$ systolic) was still raised. In both patients repeat pulmonary arteriograms at two days (Case 5) and three days (Case 6) showed complete disappearance of the previously noted filling defects.

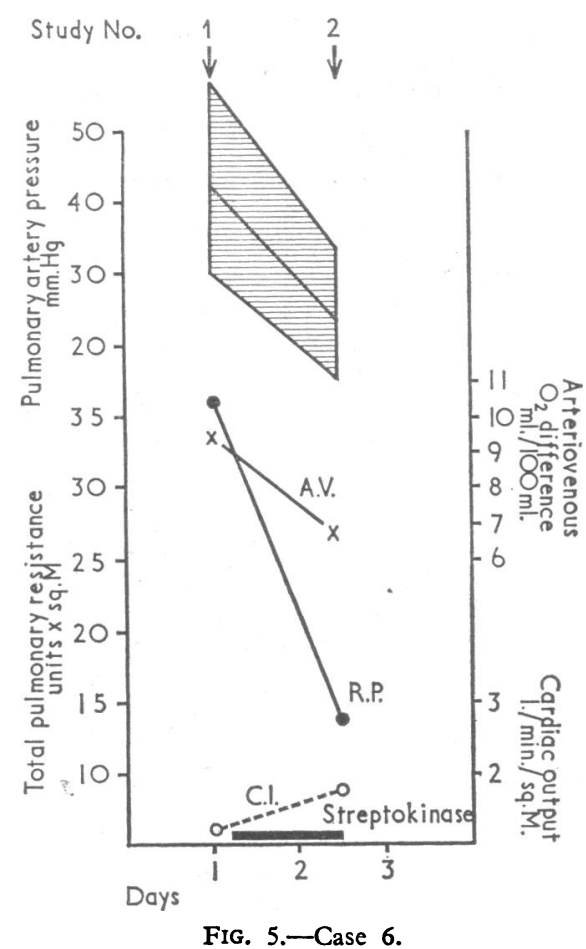

\section{Chronic Pulmonary Thromboembolism}

There were three patients with pulmonary embolism of longer duration.

Case 7.-Pulmonary arteriography performed six weeks after the embolic episode showed no major filling defects but changes compatible with relatively chronic thromboembolism affecting mainly the right pulmonary artery branches. Pulmonary artery systolic pressure was raised at $35 \mathrm{~mm}$. $\mathrm{Hg}$ systolic and was unchanged when the patient was restudied one week after starting a 36-hour infusion of streptokinase into the pulmonary artery. At this time a repeat pulmonary arteriogram showed some improvement in flow to the right lung but was still grossly abnormal.

Case 8.- This patient, an asthmatic taking steroids, had a history of thrombophlebitis and had been increasingly breathless with haemoptysis for one month. Pulmonary arteriography showed multiple filling defects affecting the right upper and lower lobe arteries and occlusion of the left lower lobe artery. When restudied 12 days after starting a 36-hour infusion of streptokinase into an arm vein there was no significant arteriographic or haemodynamic change.

Case 9.- This patient had a history of repeated pulmonary embolism occurring over 12 years, the most recent event, for which he was treated with anticoagulants, occurring two months before transfer to this hospital. A pulmonary arteriogram at the time of admission to this hospital showed complete occlusion of the left pulmonary artery as well as some filling defects in segmental branches of the right lower lobe pulmonary artery. Pulmonary artery pressure was raised $(36 \mathrm{~mm}$. $\mathrm{Hg}$ systolic) and rose to $65 \mathrm{~mm}$. $\mathrm{Hg}$ systolic on mild exercise. At operation old clot was removed from the left pulmonary artery, but two weeks postoperatively a repeat study showed no change in the arteriographic appearances. It was assumed that thrombosis had occurred on the roughened intima at the site of the previous embolus. Since this thrombus was not more than two weeks old streptokinase was infused into the pulmonary artery for 36 hours. Three days after starting treatment arteriographic appearances were still unchanged and pulmonary artery pressure was $60 \mathrm{~mm}$. $\mathrm{Hg}$ systolic.

\section{Discussion}

Probably some two-thirds of all patients dying from massive pulmonary embolism do so within two hours of the acute episode (Gorham, 1961); such patients are not represented in the present series, and it is unlikely that streptokinase will play a part in their treatment. Choosing the correct treatment for those patients with massive pulmonary embolism who survive for more than two hours and those with minor emboli is made difficult by lack of knowledge of the natural history of the disease and, more importantly, by inability to predict the outcome, in any particular patient, at the time of the acute event (Lancet, 1966; Thomas, 1965). Although spontaneous resolution of the pulmonary emboli has been shown in man (Fred et al., 1966) and in animals (Allison et al., 1960), patients undoubtedly exist in whom such resolution does not occur (Goodwin et al., 1963) and Case 9 reported here is just such a patient.

The value of heparin therapy in pulmonary embolism has been emphasized (Barritt and Jordan, 1960); however, Tow and Wagner (1967), using lung scans to determine the rate of recovery in 69 patients with pulmonary emboli treated with anticoagulants, found that of 11 with severe or massive embolism complete recovery could be shown in only one (at the 60th day) and the earliest partial recovery was observed on the eighth day. Moreover, these authors, while confirming complete recovery in many patients, found that even in the group with minimal involvement recovery was complete in only $50 \%$ at the end of one month. Hirsh et al. (1967) reported the case of a patient in whom little arteriographic change was observed after 24 hours' treatment with heparin, but in whom a subsequent 24-hour infusion of streptokinase resulted in a striking improvement. This patient is similar to Case 2 reported here in whom heparin had been given for six days without haemodynamic or angiographic improvement but in whom resolution was complete 48 hours after starting a subsequent 36 -hour infusion of streptokinase. While such results suggest that streptokinase therapy may lead to more rapid and complete resolution of pulmonary emboli than occurs with anticoagulants or by natural lytic mechanisms alone, firm conclusions must await a properly controlled trial of such therapy.

Despite the reported successful use of streptokinase in arterial thrombosis as long ago as 1962 (Cotton et al., 1962) there have been few reports of its use in pulmonary embolism. Browse and James (1964) reported the cases of four patients with pulmonary embolism who were thought on clinical grounds to have improved with streptokinase, and Hirsh et al. (1967) reported the successful treatment of massive pulmonary embolism in two patients in whom the severity of embolization and the efficacy of treatment was established by pulmonary arteriography. Dickie et al. (1967) reported two cases treated with another fibrinolytic agent, urokinase, in which pulmonary arteriograms showed complete resolution of massive emboli at nine and six days respectively. Hirsh et al. (1968) reported a larger series of 18 patients treated with streptokinase. Both these authors and Dickie and associates conclude that fibrinolytic therapy resulted in a more rapid resolution of emboli than would have been expected from the natural lytic mechanisms alone.

At the present time the place of streptokinase in the treatment of pulmonary embolism has still to be defined. It is likely that streptokinase therapy will be of most benefit in recent massive embolism (Hirsh et al., 1968), and we report four cases of massive pulmonary embolism in which streptokinase resulted in satisfactory resolution and the patients were thus spared embolectomy. However, it is likely that there will remain a proportion of patients in whom the delay in response associated with streptokinase therapy is unacceptable and in whom embolectomy may be life-saving.

Our experience of streptokinase treatment in patients with pulmonary embolic disease of longer duration has been dis- 
appointing, and it seems unlikely that streptokinase will play an important part in the treatment of such patients (Hirsh et al., 1968). Finally, while we obtained rapid resolution of minor recent emboli in two patients it must remain arguable whether any treatment, carrying with it the risk of haemorrhagic complications, is justifiable in such patients.

\section{REFERENCES}

Allison, P. R., Dunnill, M. S., and Marshall, R. (1960). Thorax, 15, 273. Barritt, D. W., and Jordan, S. C. (1960). Lancet, 1, 1309.

British Medical fournal, 1968, 4, 133.

Browse, N. L., and James, D. C. O. (1964). Lancet, 2, 1039.

Cotton, L. T., Flute, P. T., and Tsapogas, M. J. C. (1962). Lancet, 2, 1081
Dickie, K., de Groot, W., Cooley, R., Guest, M. M., and Bond, T. (1967) Tex. Rep. Biol. Med., 25, 613 .

Fred, H. L., Axelrad, M. A., Lewis, J. M., and Alexander, J. K. (1966). 7. Amer. med. Ass., 196, 1137.

Goodwin, J. F., Harrison, C. V., and Wilcken, D. E. L. (1963). Brit. Med. F., 1, 701.

Gorham, L. W. (1961). Arch. intern. Med., 108, 8.

Hirsh, J., Hale, G. S., McDonald, I. G., McCarthy, R. A., and Cade, J. F. (1967). Lancet, 2, 593.

Hirsh, J., Hale, G. S., McDonald, I. G., McCarthy, R. A., and Pitt, A. (1968). Brit. med. F., 4, 729.

Lancet, 1966, 2, 1169.

Scholander, P. F. (1947). F. Biol. Chem., 167, 235.

Thomas, D. P. (1965). New Engl. 7. Med., 273, 885.

Tow, D. E., and Wagner, H. N. (1967). New Engl. F. Med., 276, 1053. Medicine, 276, 1053.

Zijlstra, W. G., and Mook, G. A. (1962). Medical Reflection Photometry, V.an Gorcum, Assen, The Netherlands.

\title{
Value of Routine Cardiac Monitoring in the Management of Acute Myocardial Infarction outside a Coronary Care Unit
}

\author{
P. J. B. HUBNER,*§ M.B., M.R.C.P. ; M. J. GOLDBERG,† M.B., DIP.MED., M.R.C.P. ; C. W. LAWSON, $\ddagger$ M.D., F.R.C.P.
}

Brit. med. F., 1969, 1, 815-817

$S^{\text {u }}$ mmary : In a coronary care unit patients and electrocardiographic monitors are under almost continuous observation by trained personnel. This paper suggests that in a general medical ward without this facility routine cardiac monitoring with E.C.G. oscilloscopes is unlikely to lower the overall mortality from acute myocardial infarction. A mortality of $25 \%$ for acute myocardial infarction was the same for a hospital without a coronary care unit where monitoring was routinely performed and for two neighbouring hospitals which did not routinely use monitoring during the period of analysis.

The need to train personnel in the recognition of E.C.G. monitor tracings and the difficulties associated with monitor alarm systems are emphasized.

\section{Introduction}

Treatment of patients in coronary care units has been shown to lower the mortality from acute myocardial infarction (Fluck et al., 1967 ; Lawrie et al., 1967 ; Lown et al., 1967 ; Restieaux et al., 1967). This paper attempts to assess the value of routine cardiac monitoring with E.C.G. oscilloscopes in a hospital ward, without the full facilities of a coronary care unit. The results of this management are compared with those from two series of patients admitted to general hospital medical wards, where monitoring was not routinely used during the period analysed. The severity of infarction was determined to discover whether the three management groups were comparable.

\section{Material and Methods}

\section{Hospitals}

Admissions for acute myocardial infarotion to three Leicester hospitals were studied. At Groby Road Hospital, which is a regional cardiac unit, both male and female patients were admitted to the same ward, consisting of cubicles for one or two patients opening on to a long corridor. All patients with myocardial infarction were monitored for the first 48 hours, or longer if necessary. There was no coronary care unit on the ward. Two to four patients, though not always with myocardial infarction, were usually being monitored at any one time. The sisters and staff nurses were acquainted with the normal electrocardiographic (E.C.G.) rhythm pattern ; they had experience at the recognition on the monitor of traces showing extrasystoles, bradycardia, heart block, and tachycardia. However, no formal course of instruction on E.C.G. arrhythmia patterns had been given to them. Arrhythmias were actively sought for by medical and nursing staff.

At the other two hospitals-the Leicester General Hospital and the Leicester Royal Infirmary-patients with acute myocardial infarction were admitted to general medical wards and were not routinely monitored. The Leicester Royal Infirmary has an intensive therapy ward which in 1966 cared for drug poisoning $(25 \%)$, trauma $(33 \%)$, postoperative patients $(13 \%)$, myocardial infarction (6\%), and other medical conditions $(23 \%)$. The small propontion of patients with myocardial infarction admitted to intensive therapy ward were either severe cases or relatively young patients, and were usually monitored. Because their number was small-that is, 20 out of 162 myocardial infarction patients-and as no significant features were found on their analysis, they have not been separated from the Leicester Royal Infirmary figures.

All cases of acute myocardial infarction admitted to these hospitals over a 13-month period were analysed. The series consists of 102 patients admitted to Groby Road Hospital from 1 May 1966 to 31 May 1967 and 147 and 162 patients admitted to the Leicester General Hospital and the Leicester Royal Infirmary respectively, from 1 December 1965 to 31 December 1966. The later period was chosen for Groby Road Hospital so that six months' experience with monitoring equipment had

\footnotetext{
* Senior House Officer.

+ Consultant Cardiologist

Consultant Physician.

Department of Cardiology, The Groby Road Hospital, Leicester.

$\checkmark$ Present address: Department of Medicine, Hammersmith Hospital, London W.12.
} 